\title{
The Impact of Providing a Tool Kit for Innovators in an Academic Medical Center to Scale Digital Health Innovation
}

Ritika Saxena $^{1,2^{*}}$; Josephine Elias ${ }^{1 *}$, MBA, MPH; Chenzhe Cao ${ }^{1 *}$, MPH; Haipeng Zhang ${ }^{1 *}$, MMSc, DO; Adam Landman $^{1 *}$, MD, MHS, MIS, MS

\author{
${ }^{1}$ Brigham and Women's Hospital, Brigham Digital Innovation Hub, Boston, MA, United States \\ ${ }^{2}$ Brandeis University, Waltham, MA, United States \\ *all authors contributed equally
}

\section{Corresponding Author:}

Ritika Saxena

Brigham and Women's Hospital

Brigham Digital Innovation Hub

60 Fenwood Road

Boston, MA,

United States

Phone: 5999146

Email: rsaxena@brandeis.edu

\begin{abstract}
Background: An internal iHub survey shows that $72 \%$ of innovators within Academic Medical Centers abandon their ideas due to a lack of direction for their visions. While internal innovators are frustrated without direction and support to launch their ideas, hospitals need to balance innovation while ensuring information security-HIPPA compliance. Brigham and Women's Hospital houses a digital innovation hub (iHub) that fosters innovation for Brigham clinicians, scientists, researchers, administrators, and staff. In 2014, BWH founded a program called Digital Health Innovation Guide (DHIG) to provide structure for innovators to pilot new and novel technology in a safe, efficient, and successful manner. As a continuous cycle of innovation, the iHub identified successes and ways to improve the DHIG process and quality of service.
\end{abstract}

Objective: We gathered and analyzed data from participants of the DHIG and creators of the program to project the outcomes of the Digital Health Innovation Guide. With that information, we were able to quantify the impact of providing these resources and determine ways to improve the process of helping scale and structure digital health innovation.

Methods: We conducted a case review of existing data on DHIG projects. This included gathering data on projects from 2014-current. We reached out to 40 participants that went through the DHIG program to fill out a survey of questions regarding logistics of their project, successes and failures they faced, their thoughts on DHIG process, and its impact on the piloting process. We interviewed 10 participants to discuss the impact of the DHIG process, and to quantify where more support is needed from the iHub to better aid innovators to utilize and innovate new technologies in health care.

Results: From the responses collected, $50 \%$ of the innovators collaborated with external startups, while the other $50 \%$ were custom developments. $86 \%$ of teams had over 4 members, and of the remaining $14 \%$, only $20 \%$ were still actively working to pilot completion. Conversely, $100 \%$ of stalled projects had less than 4 members. Participants listed that upholding deadlines and maintaining communication with internal stakeholders as well as external, such as developers and other hospitals, brought on successes for their project. Internal bottlenecks like indeterminate delays of IRB approval timelines and info sec reviews slowed down progress and, in some cases, led to withdrawal from sponsors.

Conclusions: Based off team sizes and member engagement, we found that it is crucial to have a team of at least 4 members with an engaged clinical champion, administrative champion, and project manage to ensure pilot completion. The iHub and DHIG process can improve pilot completion by expanding external support resources such as developers and other hospitals. The DHIG, while successful in providing a clear and rigid structure for innovators in an AMC to further develop their innovations, must continue to breakdown internal barriers by acting as an expediter.

(iproc 2018;4(2):e11808) doi: $\underline{10.2196 / 11808}$ 


\section{KEYWORDS}

implementation; innovation; innovation adoption process; digital health; startups; scaling innovation; pilot

Edited by T Hale; this is a non-peer-reviewed article. Submitted 02.08.18; accepted 29.08.18; published 17.09.18.

Please cite as:

Saxena R, Elias J, Cao C, Zhang H, Landman A

The Impact of Providing a Tool Kit for Innovators in an Academic Medical Center to Scale Digital Health Innovation iproc 2018;4(2):e11808

URL: http://www.iproc.org/2018/2/e11808/

doi: $\underline{10.2196 / 11808}$

PMID:

CRitika Saxena, Josephine Elias, Chenzhe Cao, Haipeng Zhang, Adam Landman. Originally published in Iproceedings (http://www.iproc.org), 17.09.2018. This is an open-access article distributed under the terms of the Creative Commons Attribution License (https://creativecommons.org/licenses/by/4.0/), which permits unrestricted use, distribution, and reproduction in any medium, provided the original work, first published in Iproceedings, is properly cited. The complete bibliographic information, a link to the original publication on http://www.iproc.org/, as well as this copyright and license information must be included. 